\title{
Atuação do psicólogo na assistência a mulher vítima de violência doméstica: uma revisão integrativa
}

\author{
Practice of the psychologist in care for women victims of domestic violence: an integrative review \\ La práctica del psicólogo en la atención de mujeres víctimas de violencia doméstica: una revisión \\ integradora
}




\author{
Jeslaine da Silva Alves Viana \\ ORCID: https://orcid.org/0000-0003-2430-3746 \\ Centro Goiano de Ensino, Pesquisa e Pós, Brasil \\ E-mail: Jeslaine.enfermeira@gmail.com \\ Maria de Fátima Meneses da Silva \\ ORCID: https://orcid.org/0000-0002-7058-2312 \\ Centro Universitário de Ciências e Tecnologia do Maranhão, Brasil \\ E-mail: fatimacipa@ outlook.com \\ Laellya Larhanna Lima Santos Gonçalves \\ ORCID: https://orcid.org/0000-0002-4753-5593 \\ Centro Universitário de Ciências e Tecnologia do Maranhão, Brasil \\ E-mail: laellyalima@gmail.com \\ Sylmara Emanuela Rocha dos Santos \\ ORCID: https://orcid.org/0000-0002-1205-7691 \\ Centro Universitário de Ciências e Tecnologia do Maranhão, Brasil \\ E-mail: sylmararocha4@gmail.com \\ Valdileno Silva Mendes \\ ORCID: https://orcid.org/0000-0001-6295-0122 \\ Centro Universitário de Ciências e Tecnologia do Maranhão, Brasil \\ E-mail: valdilenomendes2@gmail.com \\ Dionatan de Deus Cunha Sousa \\ ORCID: https://orcid.org/0000-0001-9342-2218 \\ Faculdade Unileya, Brasil \\ E-mail: dedeus87@gmail.com \\ Francileide da Silva Sousa \\ ORCID: https://orcid.org/0000-0001-8141-1490 \\ Centro Universitário FAVENI, Brasil \\ E-mail: leda18anos@gmail.com \\ Mayra Ramalho de Sousa Rêgo \\ ORCID: https://orcid.org/0000-0003-4704-8645 \\ Centro Universitário de Ciências e Tecnologia do Maranhão, Brasil \\ E-mail: mayraramalhoofc3@gmail.com \\ Pedro Henrique Medeiros de Andrade \\ ORCID: https://orcid.org/0000-0003-1819-9540 \\ Centro Universitário de Ciências e Tecnologia do Maranhão, Brasil \\ Email: pedro.medeiroscx@gmail.com \\ Pedro Wilson Ramos da Conceição \\ ORCID: https://orcid.org/0000-0002-3868-4917 \\ Centro Universitário de Ciências e Tecnologia do Maranhão, Brasil \\ E-mail: pedrowilsonramos@gmail.com
}

\title{
Resumo
}

A sociedade sempre esteve atrelada a cultura de domínio machista e memorização da mulher, onde muitas vezes, pulsões agressivas e sexuais dos homens são saciadas utilizando a violência e agressão no ambiente intrafamiliar, neste aspecto o objetivo da pesquisa foi investigar na literatura científica como se dá a atuação do psicólogo frente a mulheres vítimas de violência doméstica. O presente estudo tratou-se de uma pesquisa bibliográfica do tipo revisão integrativa da literatura. Quanto aos resultados, Estudos têm demonstrado que a violência doméstica prejudica a saúde reprodutiva, física, principalmente emocional e mental das mulheres, onde compreender o fenômeno é necessário para o acompanhamento integral das mulheres, principalmente psicológico. O estigma e o medo de serem julgados mobilizam sentimentos de perseguição e fazem com que as vítimas considerem seu ambiente social como acusatório, como se a sociedade fosse culpá-las pela violência que sofreram, como resultado, algumas mulheres acreditam que são responsáveis pela violência e acreditam que poderiam ter evitado. Conclui-se que, o presente estudo permitiu conhecer mais sobre a atuação do psicólogo e a assistência à mulher vítima de violência doméstica e suas intervenções, com o objetivo de prevenir ou eliminar os sintomas e promover a reintegração da vítima na sociedade, além disso, incentivar as vítimas a pedir ajuda de forma antecipada, antes que a situação se agrave, resultando em uma violência física.

Palavras-chave: Psicologia; Violência doméstica; Sistemas de apoio psicossocial.

\section{Abstract}

Society has always been linked to the culture of sexist dominance and women's memorization, where often, men's aggressive and sexual drives are satiated using violence and aggression in the intra-family environment. the role of the psychologist in relation to women victims of domestic violence. The present study was a bibliographic research of the integrative literature review type. As for the results, Studies have shown that domestic violence harms the reproductive, physical, especially emotional and mental health of women, where understanding the phenomenon is necessary for the integral monitoring of women, especially psychological. The stigma and fear of being judged mobilize feelings of persecution and make victims consider their social environment as accusatory, as if society were 
to blame them for the violence they suffered, as a result, some women believe they are responsible for the violence and believe they could have avoided it. It is concluded that this study allowed us to know more about the role of psychologists and assistance to women victims of domestic violence and their interventions, with the aim of preventing or eliminating the symptoms and promoting the victim's reintegration into society. encourage victims to ask for help in advance, before the situation escalates, resulting in physical violence.

Keywords: Psychology; Domestic violence; Psychosocial support systems.

\section{Resumen}

La sociedad siempre ha estado vinculada a la cultura del dominio sexista y la memorización de las mujeres, donde a menudo, los impulsos agresivos y sexuales de los hombres se sacian con la violencia y la agresión en el entorno intrafamiliar. El papel del psicólogo en relación con las mujeres víctimas de violencia doméstica. El presente estudio fue una investigación bibliográfica del tipo revisión integradora de la literatura. En cuanto a los resultados, estudios han demostrado que la violencia intrafamiliar perjudica la salud reproductiva, física, especialmente emocional y mental de las mujeres, donde la comprensión del fenómeno es necesaria para el seguimiento integral de las mujeres, especialmente psicológico. El estigma y el miedo a ser juzgadas movilizan sentimientos de persecución y hacen que las víctimas consideren su entorno social como acusatorio, como si la sociedad las culpara por la violencia que sufrieron, por lo que algunas mujeres se creen responsables de la violencia y creen que podría haberlo evitado. Se concluye que este estudio permitió conocer más sobre el rol de los psicólogos y la atención a las mujeres víctimas de violencia intrafamiliar y sus intervenciones, con el objetivo de prevenir o eliminar los síntomas y promover la reinserción social de la víctima. Ayude con anticipación, antes de que la situación se agrave, lo que resultará en violencia física.

Palabras clave: Psicología; Violencia doméstica; Sistemas de apoyo psicosocial.

\section{Introdução}

A sociedade sempre esteve atrelada a cultura de domínio machista e memorização da mulher, onde muitas vezes, pulsões agressivas e sexuais dos homens são saciadas utilizando a violência e agressão no ambiente intrafamiliar (Neves, Dias \& Paravidini, 2013). A violência doméstica pode ser cometida dentro e fora de casa, por qualquer integrante da família que esteja em relação de poder com a pessoa agredida. Incluindo também as pessoas que estão exercendo a função de pai ou mãe, mesmo sem laços de sangue, o que é preocupante quando se busca assegurar melhores condições as mulheres que são inferiorizadas e prejudicadas com a realidade patriarcal e machista da sociedade brasileira (Waiselfisz, 2015).

$\mathrm{Na}$ atualidade existem quatro formas mais prevalente de violência no ambiente intrafamiliar, são elas: física, psicológica, negligência e violência sexual (Galvão, 2018), onde independentemente do método ou abordagem que o psicólogo irá trabalhar é necessário que haja primeiramente um vínculo terapêutico e Rapport para que a vítima se sinta em uma ambiente mais confiável e seguro, onde o psicólogo possa trabalhar com questões como: autoestima, acolhimento, oferecer orientações, (Santini\& Willams, 2018).

A prevalência da violência contra a mulher no Brasil persiste e tem demonstrada a falta de maior vigor no que tange leis e penas mais rigorosas para a punição dos agressores, o que os deixa compelidos a praticar a violência tanto no ambiente doméstico, quanto em outros ambientes. Desta forma, surgiu a necessidade de se buscar compreender melhor a temática, onde o estudo teve como problemática "Quais evidências cientificas apontam as contribuições do Profissional de Psicologia na assistência a Mulher Vítima de Violência Doméstica?”. Para tal, a pesquisa teve como objetivo investigar na literatura científica como se dá a atuação do psicólogo frente a mulheres vítimas de violência doméstica.

\section{Metodologia}

O presente estudo tratou-se de uma pesquisa bibliográfica do tipo revisão integrativa da literatura, na qual utilizou-se a metodologia proposta por Alves e Oliveira (2016). Este procedimento foi escolhido por possibilitar a síntese e análise do conhecimento científico já produzido sobre o tema "Atuação do psicólogo na assistência a mulher vítima de violência doméstica: uma revisão integrativa". 
A revisão da literatura abrangente inclui uma análise extensa da literatura, o que ajuda a discutir métodos e resultados de pesquisas e refletir sobre pesquisas futuras. O objetivo original deste método de pesquisa foi aprofundar a compreensão de um determinado assunto com base em pesquisas anteriores. Este método fornece uma combinação de dados da literatura teórica e empírica, para adquirir um melhor entendimento do tema de interesse (Sousa, Vieira, Severino, \& Antunes, 2017).

A questão norteadora desta revisão integrativa da literatura foi "Quais evidências cientificas apontam as contribuições do Profissional de Psicologia na assistência a Mulher Vítima de Violência Doméstica?’. Para a realização da coleta de dados bibliográficos foi utilizado os Descritores em Ciências da Saúde (DECS) que disponibilizam um vocabulário estruturado nos idiomas inglês, espanhol e português, onde os descritores escolhidos foram: Psicologia; Violência Doméstica; Sistemas de Apoio Psicossocial.

A elaboração incluiu a identificação do tema e seleção da questão de pesquisa, onde foram selecionados 7 artigos acadêmicos ao final da aplicação de filtros e leitura dos mesmos. O estudo teve como critérios de inclusão, artigos publicados de janeiro de 2010 a agosto de 2020, nas línguas portuguesas que contribuíram para a análise da revisão com temáticas relacionadas aos descritores. As bases de dados utilizadas foram o Google Acadêmico, Index Psicologia - Periódicos técnicocientíficos, SciELO, Lilacs e Pepisic.

Evidencia-se que a realização de uma revisão integrativa em busca de literatura pertinente e atualizada sobre a temática em questão, exige os mesmos padrões de rigor, clareza e replicação utilizados em estudos primários, tornando-se assim, uma ferramenta importante da Prática Baseada em Evidências (PBE), pois sua condução é pautada em questões de pesquisa e em métodos explícitos para identificação, análise e síntese da literatura relevante (Fernandes \& Galvão, 2013).

\section{Resultados e Discussão}

A apresentação dos resultados está organizada em duas partes. A primeira está relacionada com a caracterização dos estudos, os artigos inclusos na pesquisa, já a segunda, relaciona-se ao cumprimento do objetivo do estudo, que diz respeito à análise da produção científica acerca da atuação do psicólogo na assistência a mulher vítima de violência doméstica.

Foram selecionados para este estudo sete (07) artigos, que abordaram a temática com visões diferenciadas de diversos autores e abordagens, porém, todos trouxeram inovações científicas adequadas, corroborando para a proposta do trabalho, como mostra a Figura 1. 
Figura 1. Fluxograma da seleção dos artigos.
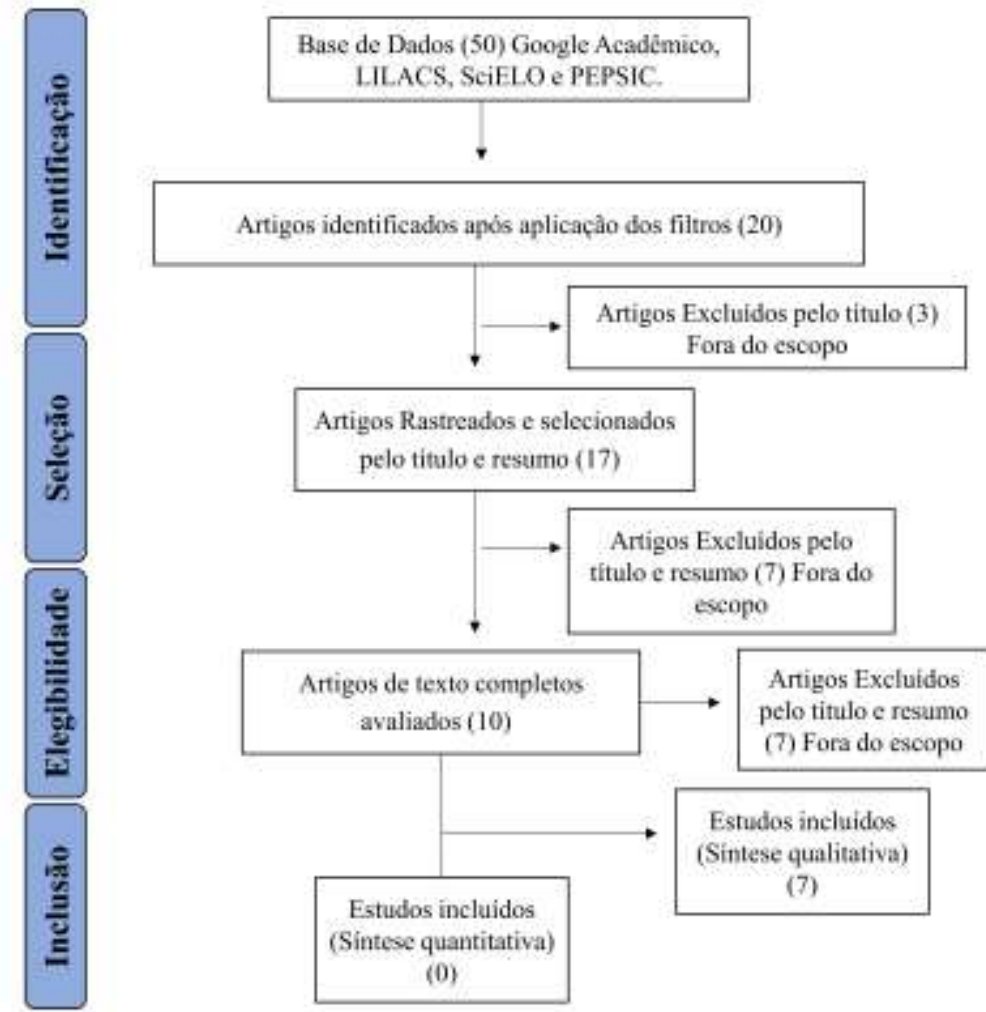

Fonte: Adaptação de Moher, Liberati, Tetzlaff e Altman (2009).

De acordo com a Figura 1 anterior foram selecionados e inclusos, artigos qualitativos com revisões de literatura com informações relevantes, no qual não se aproximasse de forma mais enfática do tema abordado, como mostra a tabela a seguir.

Tabela 1: Síntese dos resultados encontrados por artigo.

\begin{tabular}{|c|c|c|c|c|c|}
\hline $\mathbf{N}^{\circ}$ & Autor/Ano & Base de Dados & Objetivo Central & Método & Conclusões \\
\hline 1 & $\begin{array}{l}\text { Trentin et al. } \\
\text { (2018) }\end{array}$ & LILACS & $\begin{array}{l}\text { Refletir como o psicólogo, junto com outros } \\
\text { profissionais, realiza a abordagem a mulheres } \\
\text { em situação de violência doméstica. }\end{array}$ & $\begin{array}{l}\text { Pesquisa de natureza } \\
\text { qualitativa, de abordagem } \\
\text { exploratória e analítica. }\end{array}$ & $\begin{array}{c}\text { Atuação do psicólogo com a equipe } \\
\text { multidisciplinar na perspectiva da } \\
\text { bioética. }\end{array}$ \\
\hline 3 & $\begin{array}{l}\text { Campos, } \\
\text { Tchalekian \& } \\
\text { Paiva }(2020)\end{array}$ & SCIELO & $\begin{array}{l}\text { Identificar os desafios da assistência à } \\
\text { violência contra a mulher, dentro da pandemia. }\end{array}$ & $\begin{array}{l}\text { Pesquisa - Intervenção } \\
\text { com entrevistas } \\
\text { semiestruturada. }\end{array}$ & $\begin{array}{c}\text { Aprimorar formas de atendimento e } \\
\text { acessibilidade para mulheres vítimas } \\
\text { de violência doméstica dentro da } \\
\text { pandemia. }\end{array}$ \\
\hline 4 & $\begin{array}{l}\text { Belarmino, } \\
\text { Silva, Santos } \\
\text { \& Dimenstein } \\
\quad(2020)\end{array}$ & LILACS & $\begin{array}{l}\text { Refletir a importância das políticas públicas } \\
\text { fortalecendo seu funcionamento. }\end{array}$ & $\begin{array}{c}\text { Pesquisa bibliográfica e } \\
\text { qualitativa }\end{array}$ & $\begin{array}{l}\text { Aprimorar as políticas públicas de } \\
\text { forma mais abrangente. }\end{array}$ \\
\hline 5 & Paixão (2012) & LILACS & $\begin{array}{l}\text { Analisar a vivência da violência nas relações } \\
\text { conjugais }\end{array}$ & $\begin{array}{c}\text { Pesquisa descritiva e } \\
\text { qualitativa }\end{array}$ & $\begin{array}{c}\text { Identificar ações de cunho educativo } \\
\text { para prevenção da violência } \\
\text { doméstica }\end{array}$ \\
\hline 7 & $\begin{array}{l}\text { Macarini \& } \\
\text { Miranda } \\
\text { (2018) }\end{array}$ & PEPSIC & Identificar formas de intervenção psicológica. & $\begin{array}{c}\text { Pesquisa bibliográfica e } \\
\text { qualitativa. }\end{array}$ & $\begin{array}{l}\text { Ações que visam tanto o } \\
\text { enfrentamento como a prevenção a } \\
\text { violência. }\end{array}$ \\
\hline
\end{tabular}

Fonte: Dados de pesquisa (2020). 
O quadro acima foi incluído para a síntese interpretativa (01) artigo no SCIELO, (01) artigo no PEPSIC, (01) Index Psicologia - Periódicos técnico-científicos e (04) no LILACS, foram organizados pela tabela quanto o autor e ano, base de dados, objetivo central, métodos e conclusões.

A análise dos estudos incluídos nesta revisão permitiu a compreensão da dimensão psicológica da mulher que foi afetada pela violência e as sequelas às vezes persistem para sempre. Estudos têm demonstrado que a violência doméstica prejudica a saúde reprodutiva, física, principalmente emocional e mental das mulheres, onde compreender o fenômeno é necessário para o acompanhamento integral das mulheres, principalmente psicológico (Trentin et al., 2018).

Muitas mulheres se sentem envergonhadas, rejeitadas e caluniadas pela sociedade, família e amigos. O estigma e o medo de serem julgados mobilizam sentimentos de perseguição e fazem com que as vítimas considerem seu ambiente social como acusatório, como se a sociedade fosse culpá-las pela violência que sofreram. Como resultado, algumas mulheres acreditam que são responsáveis pela violência e acreditam que poderiam ter evitado. Neste aspecto, psicólogos envolvidos em métodos cognitivo-comportamentais usam ferramentas de avaliação objetivas (como uma lista do estresse e impacto do evento na vida de uma mulher) para avaliar o efeito do tratamento e servir como padrão de alta em psicoterapia individual (Silva \& Vagostello, 2017).

Um dos autores citou o acolhimento, como um dos principais objetivos do atendimento à violência contra as mulheres. Porém, foram constatadas fragilidades no manuseio de importantes ferramentas de trabalho (como visitas porta-aporta, escuta e acolhimento, trabalho em equipe e divulgação de serviços à comunidade). Sobre a qualidade de escuta e boasvindas, o momento do atendimento porta-a-porta merece a atenção dos profissionais, pois contém uma ferramenta única que permite aos usuários compreender verdadeiramente a real situação, as intervenções psicossociais também devem envolver famílias e comunidades para melhor monitorar a situação (Belarmino, Silva, Santos \& Dimenstein, 2020).

Diante deste cenário foi evidenciado que a elaboração de protocolos possibilita um melhor gerenciamento, pois provê um atendimento rápido e auxilia o profissional nas orientações e encaminhamentos pertinentes nos casos de violência doméstica. A psicologia também discute a importância de se estabelecer serviços públicos mais acessíveis, adequados e acolhedores que promovam a inclusão social e a recuperação humana, no caso da violência sexual, esse requisito é adequado porque as mulheres que vivenciaram ou vivenciam esse fenômeno precisam olhar além do físico, que entende todos os aspectos do ser humano e protege seus direitos. (Trentin et al., 2018; Campos, Tchalekian \& Paiva, 2020).

O atendimento psicológico muitas vezes é confundido com o trabalho do assistente social, porém, a psicologia está direcionada nos aspectos comportamentais e nas emoções das mulheres, enquanto o serviço social está direcionado às vulnerabilidades sociais, onde o psicólogo é um profissional que tem uma vasta área de atuação. Infelizmente a violência domiciliar vem ocasionando transtornos de humor, depressão, isolamento, desencadeando até o suicídio, para ajudar na autonomia e na melhora da qualidade de vida da mulher, é necessário um processo psicoterápico de orientação cognitivocomportamental, com uma intervenção estruturada e direta que possa ajudar amenizar os sintomas, o estresse e o sofrimento psíquico (Paixão, 2012).

Diante disso, a importância da atuação do psicólogo juntamente com a equipe multidisciplinar é de grande relevância, pois além de trabalhar diretamente com a vítima e seus traumas, também trabalha na reinclusão do ser humano na sociedade. O primeiro contato com a mulher é de grande importância, ajuda a estabelecer um vínculo necessário para o início do processo psicoterapêutico e de acordo com a singularidade de cada caso, a intervenção pode ser breve ou de longa duração, podendo também ser individual com duração de 6 a 12 sessões e após a melhoria dos seus sintomas, encaminhando para um grupo psicoeducativo e psicoterapia em grupo, com intuito a reintegração social, a troca de experiências e integração de elementos dissociados de experiência traumática (Macarini \& Miranda, 2018). 
A importância do conhecimento profissional é de suma importância, pois ajuda a promover intervenções e obter formas de enfrentamento da violência contra a mulher. O profissional deve ser instrumentalizado, ou seja, obter um conjunto de saberes e práticas necessárias para lidar com as vítimas, assim como o enfrentamento pessoal dos profissionais, aumentando as chances de obter ótimos resultados no seu atendimento. Dessa forma o profissional da psicologia que obtém conhecimentos necessários e facilidade para enfrentamento de acordo com a questão da paciente, terá uma melhor evolução e desempenho nas suas terapias (Nascimento, 2011).

\section{Considerações Finais}

Por fim, recomenda-se que a violência doméstica seja amplamente discutida na sociedade atual, e a discussão precisa mudar o paradigma para embasar as visões sobre o papel do homem e da mulher na família e na sociedade. Nesse sentido, intervenções e pesquisas sobre os autores de possíveis violências domésticas também são consideradas de extrema relevância. Recomenda-se ainda a realização de novas pesquisas qualitativas e quantitativas, longitudinais, com foco no estudo de mulheres em situação de violência.

O presente estudo permitiu conhecer mais sobre a atuação do psicólogo e a assistência à mulher vítima de violência doméstica e suas intervenções, com o objetivo de prevenir ou eliminar os sintomas e promover a reintegração da vítima na sociedade, além disso, incentivar as vítimas a pedir ajuda de forma antecipada, antes que a situação se agrave, resultando em uma violência física.

Neste aspecto, o profissional além de mostrar seu comprometimento e assumir sua função dentro das políticas públicas, deve se sentir seguro e não criar muita resistência ao atendimento a esses casos, pois o primeiro contato com a vítima, o acolhimento e a escuta, é de suma importância, para com que a mesma se sinta confortável em relatar o ocorrido e retorne ao atendimento.

A violência deve ser compreendida a partir de uma perspectiva mais abrangente, ressaltando seu enfoque relacional, interseccional e de gênero, no qual, o profissional de psicologia se permita ser o mais neutro possível, sem colocar suas experiências e ter uma postura ética.

\section{Referências}

Alves, D. L., \& Oliveira, F. B. M. (2016). Relação entre a sobrecarga de trabalho e erros de administração de medicação na assistência hospitalar. Revista Ciências \& Saberes, 2(2).

Belarmino, V. H., Silva, J.C. D. A. E., Santos, L. L. D. A., \& Dimenstein, M. (2020). Reflexões sobre práticas e cotidiano institucional na rede de proteção à mulher. Psicologia: Ciência e Profissão, 40, 1-13.

Campos, B., Tchalekian, B., \& Paiva, V. (2020). Violência contra a mulher: vulnerabilidade programática em tempos de sars-Cov-2/covid-19 em São Paulo. Psicologia \& Sociedade, 32, 1-20.

Da Silva, E. P., \& Vagostello, L. (2017). Intervenção psicológica em vítimas de estupro na cidade de São Paulo. Arquivos Brasileiros de Psicologia, 69(3), 183-198.

Galvão, P. (2018). Violência doméstica e familiar.

Fernandes, A. M., \& Galvão, P. R. (2016). A Controladoria como ferramenta de gestão nas micro e pequenas empresas: um estudo da viabilidade e da relação custo benefício. Revista de Tecnologia Aplicada, 5(1), 3-16.

Macarini, S. M., \& Miranda, K. P. (2018). Atuação da psicologia no âmbito da violência conjugal em uma delegacia de atendimento à mulher. Pensando familias, 22(1), 163-178.

Moher, D., Liberati, L., Tetzlaff, J., \& Altman, D. G. (2009). Preferred reporting items for systematic reviews and meta-analyses: the PRISMA statement. Plos Medicine, 6(7), e1000097.

Nascimento, E. D. F. G. A., (2011). Percepções dos profissionais de saúde de Angola sobre a violência contra a mulher na relação conjugal (Dissertação de Mestrado). Fundação Oswaldo Cruz, Rio de Janeiro, RJ, Brasil.

Neves, A. S., Dias, A. S. F., \& Paravidini, J. L. L. (2013). A psicodinâmica conjugal e a contemporaneidade. Psicol. clin., 25(2), $73-87$. 
Research, Society and Development, v. 10, n. 15, e83101522586, 2021

(CC BY 4.0) | ISSN 2525-3409 | DOI: http://dx.doi.org/10.33448/rsd-v10i15.22586

Paixão, G. P. N. (2013). Violência conjugal: compreendendo o fenômeno a partir do discurso feminino (Dissertação de Mestrado). Universidade Federal da Bahia, Salvador, BA, Brasil.

Santini, P. M., \& Williams, L. C. D. A. (2016). Efeitos de procedimentos para maximizar o bem-estar e a competência parental em mulheres vitimizadas. Estud. psicol., 33(4), 711-721.

Sousa, L. M. M., Vieira, C. M., Severino, S., \& Antunes, V. (2017). Metodologia de Revisão Integrativa da Literatura em Enfermagem. Revista Investigação em Enfermagem, 17-26.

Trentin, D., Vargas, M. A. D. O., Pires, D. E. P. D., Hell-Mann, F., Brehmer, L., \& Leal, S. C. (2018). Atención a mujeres en situación de violencia sexual desde la perspectiva de la bioética. Acta bioethica, 24(1), 117-126.

Waiselfisz, J. J (2015). mapa da violência 2015 homicídio de mulheres no brasil. Flacso. 